\title{
Fabrication of Submicrometer InP Pillars by Colloidal Lithography and Dry Etching
}

\author{
Ming-Yu Li, ${ }^{\text {a,b }}$ Shagufta Naureen, ${ }^{\text {b }}$ Naeem Shahid, ${ }^{\mathrm{b}}$ and Srinivasan Anand ${ }^{\mathrm{b}, \mathrm{z}}$ \\ ${ }^{a}$ State Key Laboratory for Modern Optical Instrumentation, Zhejiang University, Hangzhou 310027, China \\ ${ }^{b}$ School of Information and Communication Technology, Division of Semiconductor Materials, Royal \\ Institute of Technology, 16440 Kista, Sweden
}

A simple method for the fabrication of submicrometer InP pillars with large surface area coverage has been developed based on a combination of colloidal lithography and inductively coupled plasma (ICP) etching technique using $\mathrm{Cl}_{2} / \mathrm{H}_{2} / \mathrm{CH}_{4} / \mathrm{Ar}$ chemistry. Pillars with different sizes could be fabricated by using colloidal $\mathrm{SiO}_{2}$ particles with different sizes dispersed on the sample serving as masks. Pillars with lateral diameters as small as $60 \mathrm{~nm}$ and aspect ratios as high as 10:1 have been obtained. The effects of etch parameters such as radio-frequency power, ICP power, and etching time on pillar fabrication are investigated. By a suitable choice of etch parameters and utilizing erosion of colloidal (mask) $\mathrm{SiO}_{2}$ particle during etching, the height of the pillars as well as their shape can be modified from nearly cylindrical to conical shapes. Such a control on the shape of the structures in addition to the large surface coverage could be useful for applications in photovoltaics and for the fabrication of photonic crystals. For instance, continuous grading of the refractive index can be obtained for surfaces covered with conical pillars, which can be used as antireflecting surfaces in solar cells or for light extraction in light emitting diodes.

() 2010 The Electrochemical Society. [DOI: 10.1149/1.3464764] All rights reserved.

Manuscript submitted January 12, 2010; revised manuscript received June 25, 2010. Published July 23, 2010.

Colloidal lithography is an attractive method for providing micro- and nanopatterning in devices. Recently, several research works have focused on it because it provides a low cost lithography solution to produce micro- and nanopatterns with large area coverage. ${ }^{1-5}$ The potential of colloidal lithography for the fabrication of nanostructures has been noted in several application contexts such as in photonic crystals, ${ }^{6}$ biological, ${ }^{7,8}$ solar cells, ${ }^{9}$ and data storage. ${ }^{10}$ For applications such as surface texturing, a layer of selfassembled colloidal particles as a lithographic mask simplifies the mask fabrication process, which is one of the critical and timeconsuming steps in conventional photolithography or electron-beam lithography. ${ }^{2}$ The basic principle of colloidal lithography for pattern transfer is to utilize the deposited colloidal particles as etch masks. The colloidal mask material is typically more resistant to subsequent etching processes used to pattern the substrate material. In addition, the diameter of the deposited colloidal particles can be decreased by a suitable dry etching process while retaining their original position. ${ }^{11,12}$ Inverted structures (holes) can be etched into the substrate, for example, by depositing a metal film and subsequent removal of the colloidal particles. Thus a variety of structures, random or periodic, including periodic two-dimensional nanopillars ${ }^{13}$ and three-dimensional nanostructures, ${ }^{14}$ can be fabricated by colloidal lithography. Further, it could be of interest for certain applications if the shape, height, and surface density of the pillars can be modified by suitable processes and/or mask conditions. For instance, continuous grading of the refractive index can be obtained for surfaces covered with conical pillars, which can be used as antireflecting surfaces in solar cells ${ }^{15}$ or for light extraction in light emitting diodes (LEDs). Enhanced light extraction in GaN-based LEDs has recently been demonstrated by surface structuring using reactive ion etching (RIE) and colloidal lithography. ${ }^{16}$

Most of the research on surface nanostructuring of semiconductors using colloidal lithography has focused on Si. In comparison, very few works apply this method for III-V materials/devices. Etching of nanostructures in $\mathrm{GaAs}$ has been demonstrated using colloidal metal as etch masks, ${ }^{17,18}$ or by using colloidal lithography and metal deposition, ${ }^{19}$ or by using silica particles as etch masks. ${ }^{20}$ Recently, colloidal silica has been used as an etch mask to obtain antireflecting surfaces in GaSb. ${ }^{21}$ Considering the technological importance of InP-based materials for optoelectronic devices, it is attractive to develop low cost nanostructuring methods to improve device performance or to provide new functionalities. For example, nanostructuring could be beneficial for InP-based photovoltaic and near-IR

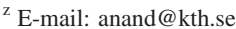

LEDs. In this paper, we demonstrate a simple method combining colloidal lithography with one-step inductively coupled plasma (ICP) etching to fabricate submicrometer InP pillars with macroscale surface coverage. Pillars with different sizes can be fabricated by using colloidal $\mathrm{SiO}_{2}$ particles with different sizes dispersed on the sample serving as masks. The effects of etch parameters such as radio-frequency (rf) power, ICP power, and etching time on the geometrical characteristics of the pillars are investigated. By a suitable choice of etch parameters and utilizing erosion of colloidal $\mathrm{SiO}_{2}$ mask particles during etching, it is possible to change not only the height of the pillars but also their shapes.

\section{Experimental}

The InP samples used in the experiments were typically 1 $\times 1 \mathrm{~cm}$ and were obtained from (100) S-doped InP epi-ready wafers. Commercially available (from Sigma-Aldrich) colloidal $\mathrm{SiO}_{2}$ particles in water were used for forming the colloidal mask on the sample and were in three different "average" diameters $(0.15,0.5$, and $1 \mu \mathrm{m})$. The samples were cleaned with standard organic solvents and the surface oxide was stripped using 50\% HF, subsequently rinsed in deionized water, and blow-dried with nitrogen. Preliminary tests were performed to identify reasonable conditions for dispersing colloidal $\mathrm{SiO}_{2}$ particles on the InP surface. Extensive investigations were not performed with respect to different surface preparation methods, spin-coating conditions, etc. Here, our objective was to obtain samples with a reasonable coverage of the particles to investigate ICP etching of the pillars. The sample with a drop of the colloidal solution was spun at different speeds (1500$4000 \mathrm{rpm}$ ) and subsequently blow-dried under nitrogen flow. Scanning electron microscopy (SEM) inspections showed very poor surface coverage, possibly due to poor wetting properties of the ascleaned surface. To improve the wetting property, the samples were additionally treated by oxygen plasma at $1 \mathrm{~kW}$ for 2 min. After this step, the surface coverage was significantly improved. SEM inspections showed that by spinning at lower speeds, a dense monolayer (with particles touching each other) coverage in large areas could be obtained. At $4000 \mathrm{rpm}$, a reasonably uniform coverage with isolated particles, albeit with small areas with dense coverage, was obtained. This allows investigation of the pillar etching under both conditions, when they are isolated or dense. Thus, for all etching results using colloidal masks discussed here, the samples were oxygen plasma treated and the spinning speed was at $4000 \mathrm{rpm}$.

Test patterns were fabricated using conventional photolithography; a $350 \mathrm{~nm}$ thick $\mathrm{SiO}_{2}$ film deposited by plasma-enhanced chemical vapor deposition (PECVD) served as the etch mask for the etching of InP. The $\mathrm{SiO}_{2}$ film was patterned by $\mathrm{RIE}$ using $\mathrm{CHF}_{3} / \mathrm{Ar}$ 


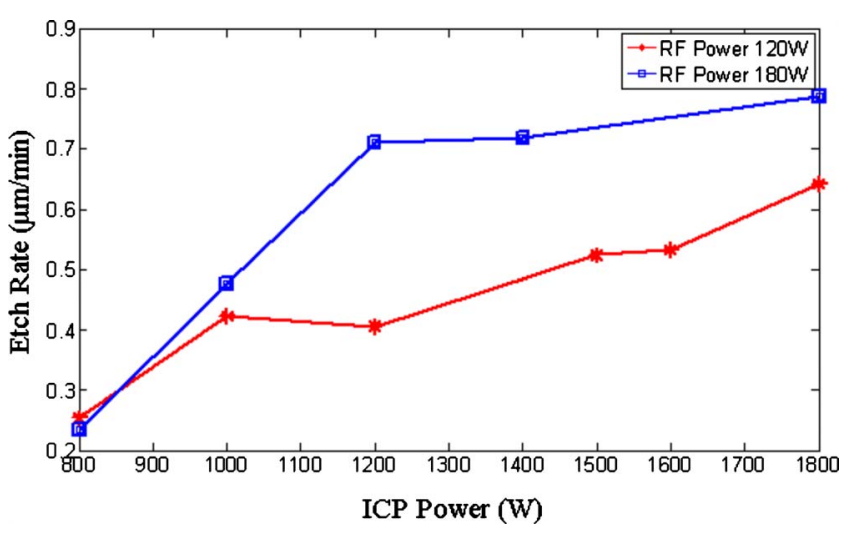

Figure 1. (Color online) Etch rate of InP as a function of ICP power at two different rf powers.

chemistry. The etching of InP was performed by ICP-RIE (Oxford Plasmalab System 100) using $\mathrm{Cl}_{2} / \mathrm{H}_{2} / \mathrm{CH}_{4} / \mathrm{Ar}$ chemistry and a piece of $\mathrm{Si}$ wafer was used as the carrier. For most of the etching experiments reported here, the flows of $\mathrm{Cl}_{2}, \mathrm{H}_{2}, \mathrm{CH}_{4}$, and $\mathrm{Ar}$ were 7, 5, 5, and $5 \mathrm{sccm}$, respectively; the operating pressure was $4 \mathrm{mTorr}$ and the sample temperature was $60^{\circ} \mathrm{C}$. For a fixed rf power (120 or 180 $\mathrm{W})$, the ICP power was varied from 800 to $2800 \mathrm{~W}$ to investigate the etch rate and etched pillar shape. SEM was used for characterizing the etched structures.

\section{Results and Discussion}

Preliminary experiments were performed with the test structures. However, the range of ICP power was restricted to $1800 \mathrm{~W}$ because the thickness of the $\mathrm{SiO}_{2}$ mask (350 nm thick) was limited. Nevertheless, it provides useful information in terms of mask selectivity and etch rates especially for colloidal masks with 150 and $500 \mathrm{~nm}$ particle sizes. Figure 1 shows the determined etch rates as a function of ICP power for two different rf powers. The etch rate can be varied from $\sim 0.25 \mu \mathrm{m} / \mathrm{min}$ up to $\sim 0.8 \mu \mathrm{m} / \mathrm{min}$; the lower etch rate can be chosen to etch shallow pillars. Figure 1 also shows that for a given ICP power $(>800 \mathrm{~W})$, the etch rate increases with rf power due to the sputter enhanced etching by increased ion energy. Over the investigated range, the selectivity of InP over the mask was typically in the range from $6: 1$ to $7: 1$. However, the selectivity could be different for the colloidal $\mathrm{SiO}_{2}$ particles compared to the PECVD deposited thin film. In addition, owing to the spherical shape of the colloidal $\mathrm{SiO}_{2}$ particles, reduction in particle (lateral) size during etching can be expected to play a strong role in determining the etch profiles. The lateral etch rate, which is important for nanostructures, could not be determined from the macrotest patterns. Below we discuss the etching of pillars using the colloidal $\mathrm{SiO}_{2}$ masks. Owing to the large particle size, samples with $1 \mu \mathrm{m}$ particles were first investigated because both the etch time and plasma powers can be increased.

Figure 2 shows the SEM pictures of the InP pillars, which were etched for $3 \mathrm{~min}$ with different ICP and rf powers The diameter of $\mathrm{SiO}_{2}$ particles (mask) is $1 \mu \mathrm{m}$. Following the results obtained earlier for the test patterns (Fig. 1), the height of the InP pillars increase with ICP and rf power. The estimated etch rates from Fig. 2a-e agree well with the data of Fig. 1. The sidewalls are smooth and nearly vertical. The SEM images also show the $\mathrm{SiO}_{2}$ particles and do not suggest any appreciable reduction in their size under these etch conditions. Correspondingly, the lateral diameter (average) of the pillars is close to $1 \mu \mathrm{m}$. Figure $2 \mathrm{~d}$ and e shows that the pillar heights (also seen from Fig. 1) are nearly similar and indicate that shallow (low aspect ratio) cylindrical structures can be etched. These results show nearly cylindrical pillars with diameters corresponding to the mask particle diameter of $1 \mu \mathrm{m}$, indicating minimal mask erosion under these etch conditions. Keeping the etch time the same and the rf
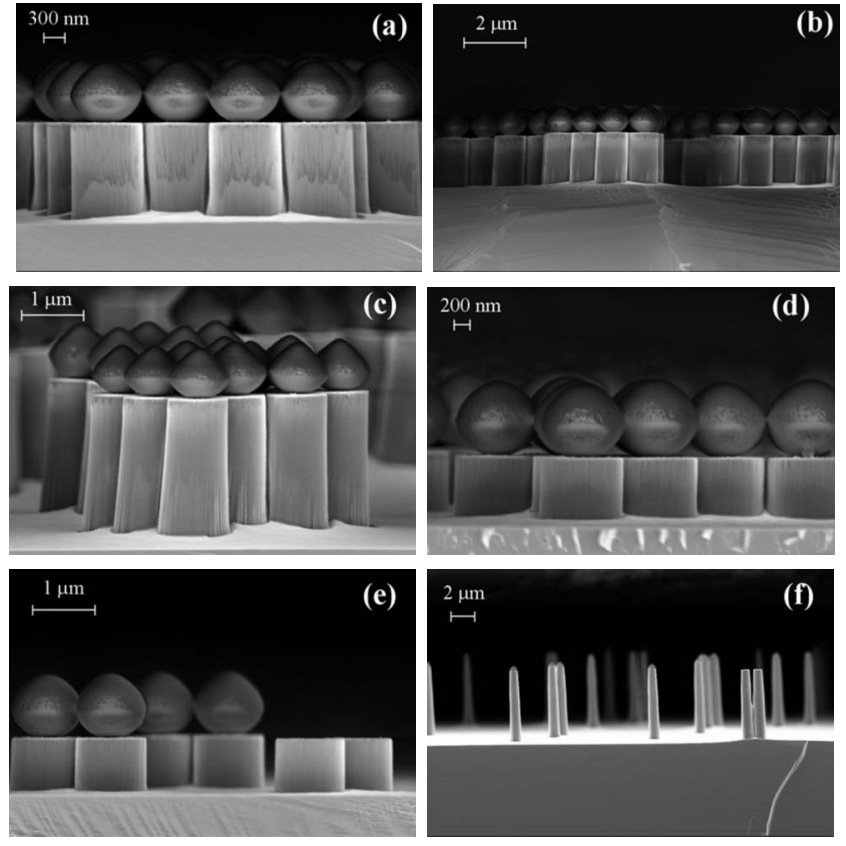

Figure 2. Cross-sectional SEM images of InP submicrometer pillars etched with different ICP and rf powers; the etch time is $3 \mathrm{~min}$ (note the different scale bars). (a) ICP $1200 \mathrm{~W}$, rf $120 \mathrm{~W}$; (b) ICP $1500 \mathrm{~W}$, rf $120 \mathrm{~W}$; (c) ICP $1800 \mathrm{~W}$, rf $120 \mathrm{~W}$; (d) ICP $800 \mathrm{~W}$, rf $120 \mathrm{~W}$; (e) ICP $800 \mathrm{~W}$, rf $180 \mathrm{~W}$; and (f) ICP $2800 \mathrm{~W}$, rf $180 \mathrm{~W}$.

power at $180 \mathrm{~W}$, the ICP power was increased to test if the shape and/or size of the pillars could be changed by mask erosion. Three etch runs were made at ICP powers of 1800, 2500, and $2800 \mathrm{~W}$. As the ICP power increased, the etch rates increased and a tendency toward tapered pillar profiles was also observed. The determined etch rates were $0.9,1.35$, and nearly $2 \mu \mathrm{m} / \mathrm{min}$ at ICP powers of 1800,2500 , and $2800 \mathrm{~W}$, respectively. The results obtained at an ICP power of $2800 \mathrm{~W}$ is shown in Fig. 2f. The obtained pillars have tapered profiles with their base diameters $(\sim 1 \mu \mathrm{m})$ corresponding to the $\mathrm{SiO}_{2}$ particle mask diameter and are $\sim 0.6 \mu \mathrm{m}$ at the top. The lateral size of the remaining mask is $\sim 0.6 \mu \mathrm{m}$, a clear reduction from the original size of $1 \mu \mathrm{m}$. These observations support the progressive erosion of the spherical mask particle and suggest that mask erosion during etching can be positively used to alter the size and shape of the pillars. At $2800 \mathrm{~W}$ (Fig. 2f), the resulting pillar heights are $\sim 6 \mu \mathrm{m}$, corresponding to an aspect ratio of 7:1. There is still $\sim 400 \mathrm{~nm}$ of $\mathrm{SiO}_{2}$ mask left, indicating a selectivity of about 1:10. This means that higher aspect ratios can be obtained by increasing the etching time.

Although the results presented above are observed for more or less isolated pillars, the etch rate and profiles of the pillars depend on their spatial density. This phenomenon is well known and is attributed to the so-called lag effect, wherein the etch rate of features with smaller openings lags behind that of structures with larger openings. A detailed investigation using samples with uniformly and densely packed monolayer of particles is necessary to fully characterize this effect and will be the subject matter of our future work. Here, in general, on the same samples, the etch rate was lower in areas with a dense particle coverage.

To investigate the suitability of this approach to fabricate smaller structures, samples with $150 \mathrm{~nm}$ colloidal $\mathrm{SiO}_{2}$ particles were used as masks. Figure 3 shows the InP nanopillars etched for two durations at ICP and rf powers of 800 and $120 \mathrm{~W}$, respectively. Assuming the mask selectivity and etch rates determined earlier, a simple estimate gives a maximum pillar height of $\sim 1 \mu \mathrm{m}$ for 3 min etching. However, the $\mathrm{SiO}_{2}$ particle also suffers lateral etching and, consequently, the effective diameters of the pillars can be reduced and the 

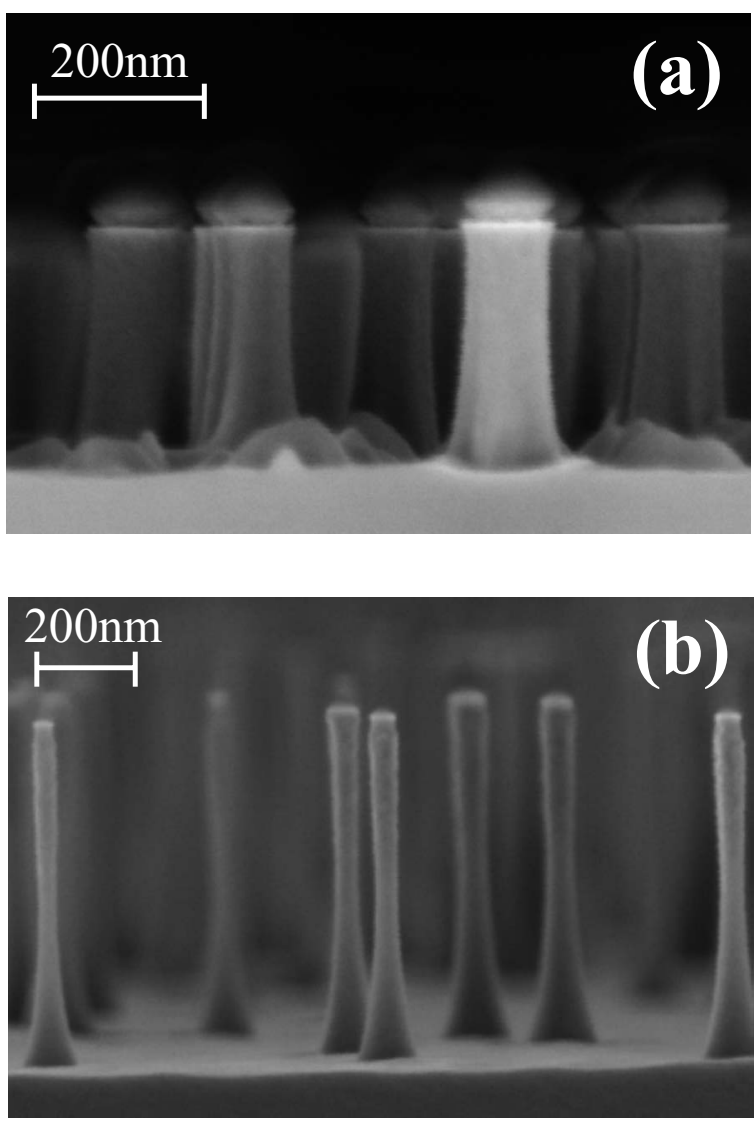

Figure 3. Cross-sectional SEM images of InP pillars etched for different durations: (a) 1 and (b) $2 \mathrm{~min}$; the ICP and rf powers are 800 and $120 \mathrm{~W}$, respectively.

mask may also disappear earlier. From Fig. 3a, even for $1 \mathrm{~min}$ etching the average lateral diameter of the pillars is reduced to $\sim 100 \mathrm{~nm}$, whereas the reduction is even more significant $(\sim 50 \mathrm{~nm}$ average diameter) for 2 min etching (Fig. 3b). The nanopillars in Fig. $3 \mathrm{~b}$ have appreciable aspect ratios of 10:1. The observed shape and lateral size of the etched pillars in Fig. 3 can be attributed to the presence of lateral etching together with mask (particle) erosion. The effect of lateral etching is more evident from the observed undercutting, which is more pronounced for a longer etch duration, as seen in Fig. 3b. Although the base diameters of the pillars in Fig. 3a are nearly equal to the mask particle size $(150 \mathrm{~nm})$, a longer etch time results in a reduction in the base diameters $(\sim 120 \mathrm{~nm})$ of the pillars (Fig. 3b).

These results from Fig. 2 and 3 suggest that by changing the etch parameters such as ICP power, rf power, and etch time, and by using erosion of the mask particle during etching, the shapes of the InP pillars can be modified. From the previous observations, for the $1 \mu \mathrm{m}$ particle masks, very long etch times may be required. In addition to the appreciable lateral etching, the smaller particle mask size (diameter: $150 \mathrm{~nm}$ ) has limited selectivity and increasing the etch time is not desirable. Thus, it seems reasonable to investigate the possibility of achieving conical pillars and of controlling their shapes by using the intermediate size for the colloidal $\mathrm{SiO}_{2}$ particles as masks (average diameter of $500 \mathrm{~nm}$ ). Compared to the $150 \mathrm{~nm}$ $\mathrm{SiO}_{2}$ particles, the $500 \mathrm{~nm}$ ones can be subjected to prolonged etching and/or to increased ICP powers, thus making it possible to progressively decrease the particle mask size during etching. Depending on the actual etching characteristics (etch rate, mask selectivity) of a given process, an appropriate particle mask size can be chosen or a separate particle mask size reduction step can be used. ${ }^{11,12}$ The results of our experiments using $500 \mathrm{~nm}$ particle masks are illus-
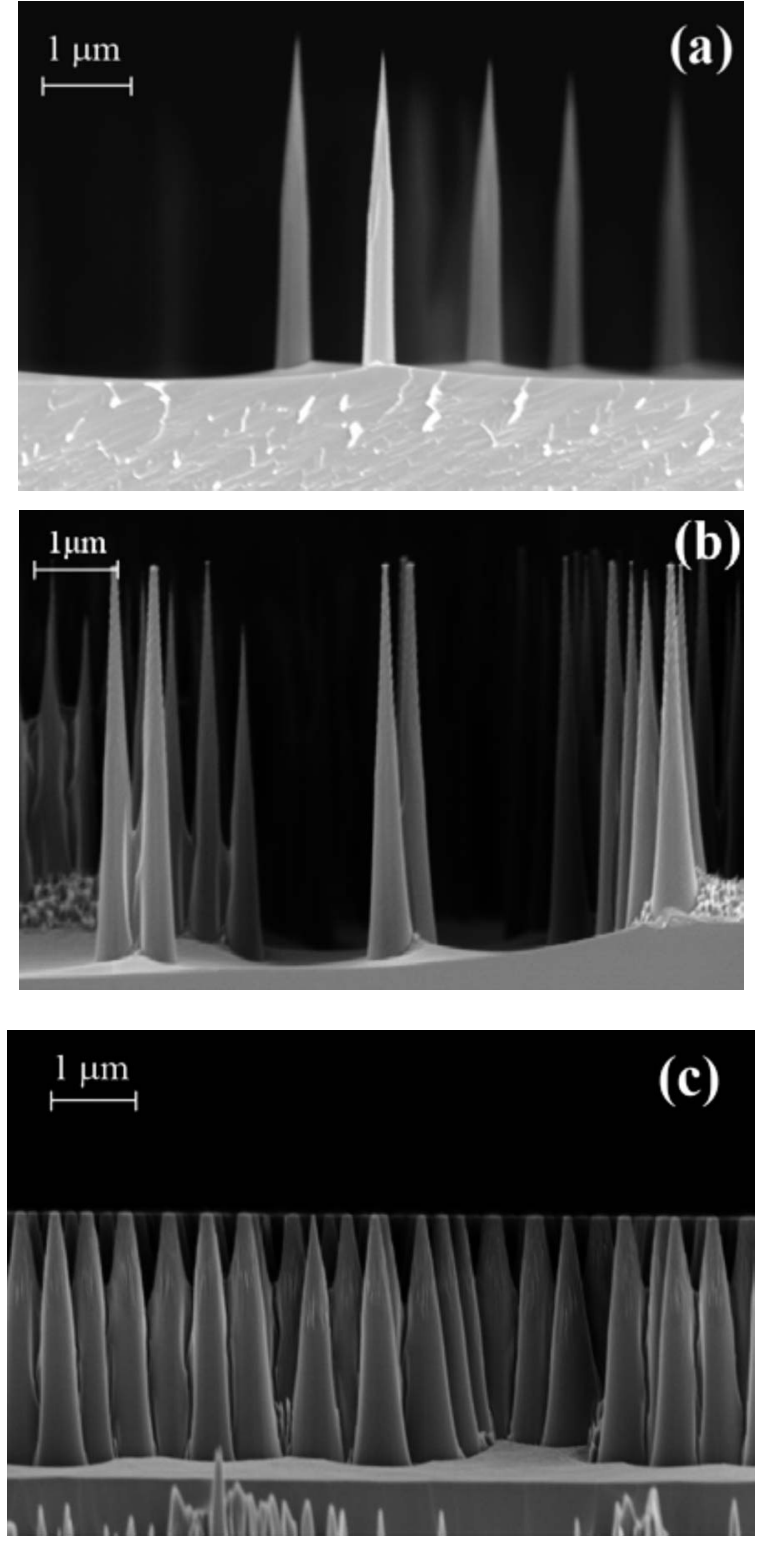

Figure 4. Cross-sectional SEM images of conical InP pillars etched at three different ICP powers: (a) 2800, (b) 2600, and (c) $2400 \mathrm{~W}$; the rf power and etch duration are $180 \mathrm{~W}$ and $3 \mathrm{~min}$, respectively.

trated in Fig. 4a-c. Figure 4 shows that the different shapes of the conical pillars can be achieved by changing the etching parameters. Here, the ICP power was varied from 2800 to $2400 \mathrm{~W}$ while keeping the rf power and etch time fixed at $180 \mathrm{~W}$ and $3 \mathrm{~min}$, respectively. For example, Fig. 4a shows the InP conical nanopillars (etched at an ICP power of $2800 \mathrm{~W}$ ) with very sharp tips, which could be useful for field-emitter applications. The bases of the pillars are typically $300 \mathrm{~nm}$ wide and suggest lateral etching in addition to mask erosion. The extremely sharp apex of the pillar suggests a complete removal of the mask. The average pillar heights are $\sim 4 \mu \mathrm{m}$, which is smaller than what was obtained under identical conditions for the $1 \mu \mathrm{m}$ mask particles (Fig. 2f). This indicates continued etching after complete erosion of the mask particle. However, the different slopes in the upper and lower portions of the pillars cannot be explained by a progressive erosion of the particle mask. It is likely to be due to a rapid removal of the mask in the later stage of the etching in combination with angle-dependent etch rate. When the ICP power is reduced, this effect is not observed; instead, conical pillars are obtained, as seen in Fig. 4b and c. In both these cases, the 
base of the pillars is nearly equal to the original particle mask diameter $(500 \mathrm{~nm})$ and it is strongly argued that the obtained shapes are primarily due to particle mask erosion. Figure $4 \mathrm{~b}$ shows the pillars etched at an ICP power of $2600 \mathrm{~W}$. The obtained pillars are conical with steep sidewalls and a sharp apex, and only a small part of the mask is left. On average, the pillar height is $\sim 4.8 \mu \mathrm{m}$, corresponding to an etch rate of $1.6 \mu \mathrm{m} / \mathrm{min}$. This is consistent with the etch rates at $2500 \mathrm{~W}(1.35 \mu \mathrm{m} / \mathrm{min})$ and $2800 \mathrm{~W}(2 \mu \mathrm{m} / \mathrm{min})$ determined using $1 \mu \mathrm{m}$ particle masks. Here, the pillar heights are not uniform and it is likely that the particle (mask) erosion rate itself could differ in different regions of the sample depending on their exact size and spatial separation. The results of Fig. 4c show that when the ICP power is reduced further to $2400 \mathrm{~W}$, conical pillars having a uniform height $(\sim 3 \mu \mathrm{m})$ are obtained. Compared to the shape of pillars etched at $2600 \mathrm{~W}$ (Fig. 4b), here the pillar tops are not as sharp and the sidewall slope is also less steep, indicating lesser erosion of the mask at $2400 \mathrm{~W}$. We recognize that angledependent etching also contributes as soon as sloped walls are formed due to mask erosion and could affect the exact slope of the sidewalls. Modeling of angle-dependent etching in the presence of mask erosion is rather complex and is beyond the scope of the present work.

Clearly, from Fig. 4b and c, conical structures can be obtained by effectively utilizing erosion of the $\mathrm{SiO}_{2}$ particle during etching. Such structures could be interesting for antireflection in photovoltaic devices and for light extraction in LEDs. For a wavelength of light longer than the average spacing of the pillars, perpendicular to the sample surface, such structures exhibit a graded refractive index varying from that of the substrate to that of air. This is one of the approaches for obtaining enhanced transmission of light. ${ }^{22}$ For light with wavelengths shorter/comparable to the average pillar spacing, diffractive effects become important. Such structures have been proposed for antireflection and may also help in enhancing the absorption of above bandgap light in the InP pillars. ${ }^{23}$ The investigation of the behavior of light in such nanostructured media is clearly of interest and will be the subject matter of our future work using samples with uniform and macroscale dense (monolayer) coverage of colloidal particles.

\section{Conclusion}

In summary, colloidal lithography and ICP etching technique can be used to create submicrometer InP pillars with a large surface area coverage. Pillars with different sizes could be fabricated by using colloidal $\mathrm{SiO}_{2}$ particles with different sizes dispersed on the sample serving as masks. Pillars with lateral diameters as small as $60 \mathrm{~nm}$ and aspect ratios as high as 10:1 have been obtained. By changing the etch parameters such as rf power, ICP power, and etch time, and by making positive use of progressive erosion of colloidal $\mathrm{SiO}_{2}$ mask particle during etching, the height of the pillars as well as their shape can be modified from nearly vertical to conical shapes. Such a control on shape of the structures in addition to the large surface coverage could be useful to produce photonic crystals, antireflection surfaces in solar cells, or for light extraction in LEDs.

\section{Acknowledgments}

This work was supported by the Swedish Research Council, the Swedish Strategic Research Foundation, the EU network of excellence Nanophotonics4energy, the National Natural Science Foundation of China (grant no. 60807018), and Zhejiang Provincial Natural Science Foundation of China (grant no. Y1090239).

Royal Institute of Technology Sweden assisted in meeting the publication costs of this article.

\section{References}

1. H. W. Deckman and J. H. Dunsmur, Appl. Phys. Lett., 41, 377 (1982)

2. H. Fredriksson, Y. Alaverdyan, A. Dmitriev, C. Langhammer, D. S. Sutherland, M. Zäch, and B. Kasemo, Adv. Mater, 19, 4297 (2007)

3. S. M. Yang, S. G. Jang, D. G. Choi, S. Kim, and H. K. Yu, Small, 2, 458 (2006)

4. J. H. Moon, W. S. Kim, J. W. Ha, S. G. Jang, S. M. Yang, and J. K. Park, Chem Commun. (Cambridge), 2005, 4107.

5. J. H. Moon, S. G. Jang, J. M. Lim, and S. M. Yang, Adv. Mater., 17, 2559 (2005)

6. V. V. Poborchii, T. Tada, and T. Kanayama, Appl. Phys. Lett., 75, 3276 (1999)

7. M. A. Wood, J. R. Soc., Interface, 4, 1 (2007).

8. M. J. Dalby, C. C. Berry, M. O. Riehle, D. S. Sutherland, H. Agheli, and A. S. G. Curtis, Exp. Cell Res., 295, 387 (2004).

9. H. L. Chen, S. Y. Chuang, C. H. Lin, and Y. H. Lin, Opt. Express, 15, 14793 (2007).

10. A. Born and R. Wiesendanger, Appl. Phys. A: Mater. Sci. Process., 68, 131 (1999).

11. T. Lohmüller, U. Müller, S. Breisch, W. Nisch, R. Rudorf, W. Schuhmann, S. Neugebauer, M. Kaczor, S. Linke, S. Lechner, et al., J. Micromech. Microeng., 18 115011 (2008)

12. C. Haginoya, M. Ishibashi, and K. Koike, Appl. Phys. Lett., 71, 2934 (1997).

13. C. W. Kuo, J. Y. Shiu, and P. Chen, Chem. Mater, 15, 2917 (2003).

14. D. G. Choi, H. O. Yu, S. G. Jang, and S. M. Yang, J. Am. Chem. Soc., 126, 7019 (2004).

15. J. Zhu, Z. Yu, G. F. Burkhard, C.-M. Hsu, S. T. Connor, Y. Xu, Q. Wang, M. McGehee, S. Fan, and Y. Cui, Nano Lett., 9, 279 (2009).

16. J. W. Park, J. H. Park, H. Y. Koo, S. I. Na, S. J. Park, H. Y. Song, J. W. Kim, W. C. Kim, and D. Y. Kim, Jpn. J. Appl. Phys., Part 1, 47, 5327 (2008).

17. P. A. Lewis, H. Ahmed, and B. W. Alphenaar, Microelectron. Eng., 57-58, 925 (2001).

18. Y. Yasukawa, H. Asoh, and S. Ono, J. Electrochem. Soc., 156, H777 (2009).

19. M. Haupt, S. Miller, A. Ladenburger, R. Sauer, K. Thonke, J. P. Spatz, S. Reithmuller, M. Moller, and F. Banhart, J. Appl. Phys., 91, 6057 (2002).

20. C.-H. Sun, B.-J. Ho, B. Jiang, and J. Peng, Opt. Lett., 33, 2224 (2008)

21. W.-L. Min, A. P. Bentancourt, P. Jiang, and B. Jiang, Appl. Phys. Lett., 92, 141109 (2008).

22. R. Windisch, C. Rooman, S. Meinlschmidt, P. Kiesel, D. Zipperer, G. H. Döhler, B. Dutta, M. Kuijk, G. Borghs, and P. Heremans, Appl. Phys. Lett., 79, 2315 (2001)

23. O. L. Muskens, J. G. Rivas, R. E. Algra, E. P. A. M. Bakkers, and A. Lagendijk, Nano Lett., 8, 2638 (2008). 